\title{
Light Trapping Limit Revisited: How do Guided Modes Enhance Light Absorption in Solar Cells?
}

\author{
Ali Naqavi ${ }^{1,2}$, Franz-Josef Haug ${ }^{1}$, Hans Peter Herzig ${ }^{2}$ and Christophe Ballif ${ }^{1}$ \\ ${ }^{1}$ Photovoltaics and Thin Film Electronics Laboratory, École Polytechnique Fédérale de Lausanne (EPFL), Breguet 2, 2000 Neuchâtel, \\ Switzerland \\ ${ }^{2}$ Optics \& Photonics Technology Laboratory, École Polytechnique Fédérale de Lausanne (EPFL), Breguet 2, 2000 Neuchâtel, Switzerland \\ e-mail:ali.naqavi@epfl.ch
}

\begin{abstract}
We explore the limit of absorption enhancement in thin film solar cells obtained by guided mode excitation and we discuss the geometrical and angular dependence of absorption. We consider the modal properties of the cell.
\end{abstract}

OCIS codes: $350.6050,040.5350,050.1950,130.2790$.

Thin film solar silicon cells are a promising choice to commercialize photovoltaics in large scale [1]. In particular, thin film silicon can benefit from the well-established flat panel display technology and its impressive achievement in cost reduction. Furthermore, the small thickness of such cells provides the potential of achieving relatively high open circuit voltage compared to thicker cells due to reduced bulk recombination. Their main deficit is their indirect band gap which reduces their light absorbability at long wavelengths.

To enhance light absorption in these cells, one should couple solar radiation into the cell more efficiently and increase its absorption before it escapes out of the cell. Different texturing methods have been applied to realize this idea and solar cells based on both random and periodic patterns have been fabricated. Nevertheless, there is a main question to respond: "How far can we go with these texturing techniques?"

The answer to the latter question depends on geometrical parameters such as the type of texture used and also on the cell thickness. For example, random "Lambertian" texturing of a weakly absorbing thick film with refractive index $n$ can increase absorption in the film by a factor of at most $4 n^{2}$ compared to the single pass absorption over the same length as the film thickness [2]. If the randomly textured film is thin, the enhancement might be less than $4 n^{2}$ depending on its thickness [3]. Periodic patterning can enhance absorption more in specific wavelength ranges because compared to the random texturing, it can couple light to preferred directions more effectively. It has been shown recently that periodic grating couplers can enhance absorption in thick solar cells by a factor of up to $8 \pi n^{2} / \sqrt{3}$ at a single wavelength if triangular geometry is used, $4 \pi n^{2}$ if the structure follows square geometry and $2 \pi n$ if one dimensional (1D) gratings are used [4, 5]. Recently, we developed a model for thin film with periodic texture and we showed that it is possible to surpass the mentioned upper bounds in limited wavelength ranges [6].

In this presentation, we investigate the upper bound of absorption in more detail. Our theory is based on the temporal coupled mode approach suggested by $\mathrm{Yu}$ et al.[4]. We apply the modal properties of the structure to distinguish the impact of different guided modes on the absorption. By considering the periodic pattern and the thin film assumptions simultaneously, our model accounts for the discrete nature of the guided modes in both spatial and temporal frequency domains. Also, we calculate the overlap of the electromagnetic energy with the absorbing region. In this way, the model introduced here can be assumed as an extension of the previous models. Thanks to the generality of the method, we also discuss the angular dependence of the absorption and the impact of the choice of grating geometry on it.

Without losing generality we consider a weakly absorbing slab of refractive index $n=4$ and thickness $d=200 \mathrm{~nm}$. We calculate the absorption enhancement under normal illumination over the wavelengths from 600 to $800 \mathrm{~nm}$ and from 800 to $1200 \mathrm{~nm}$ introduced by a 1D periodic texture with period $P=500 \mathrm{~nm}$. The choice of wavelength ranges is subject to the fact that the wavelengths from the first and the second intervals overlap the light trapping regions of amorphous silicon cells and different types of crystalline cells respectively. At each wavelength, the absorption enhancement factor $(F)$ is defined as the ratio of light absorption in the cell to the single pass absorption. Figure 1 shows $F$ as a function of normalized period $(P / \lambda)$ where $\lambda$ is the incident wavelength. It is 
observed that the maximal enhancement factor might surpass the $2 \pi n$ limit. Hence, the previous limits can be surpassed over relatively broad wavelength ranges by considering the modal properties of the structure. If $2 \mathrm{D}$ gratings are considered, similar results are obtained but the calculations are more complicated and it is the object of future investigations.

Besides the above considerations, other parameters such as the incident angle and the type of periodic pattern can change the amount of light absorption in the solar cell. The incident angle of light affects the light absorption dramatically and usually maximal absorption is expected at around perpendicular incidence. The upper bounds shown in Figure 1 were obtained for the normal incidence. Our calculations show that as the incident angle deviates from zero to angles larger than 40 degrees, the enhancement factor $F$ drops to a value around $\pi n$. Another parameter which affects the absorption in the solar cell is the shape of the grating that is used to couple light into the guided modes. It has been proposed by $\mathrm{Yu}$ et al. that gratings with a triangular lattice geometry can potentially provide more absorption than the square ones [5]. Our simulations confirm their conclusions and we also observe that the triangular pattern is less sensitive to the change of the incident angle. Note that in this manuscript and in the related previous research [2-6], the absorption enhancement is associated with the number of guided modes. Obtaining more realistic values requires one also to consider the efficiency of light coupling to the guided modes of the cell and remains object of future research.

In conclusion, we show that by benefiting from the modal structure of the solar cell, it is possible to obtain higher absorption enhancement than the previous predictions. By a detailed explanation of the temporal coupled mode theory and its application to thin film solar cells, we find guidelines for the photonic design such that the guided modes introduce more light absorption. This extends the applicability of our analysis to real solar cell design applications.
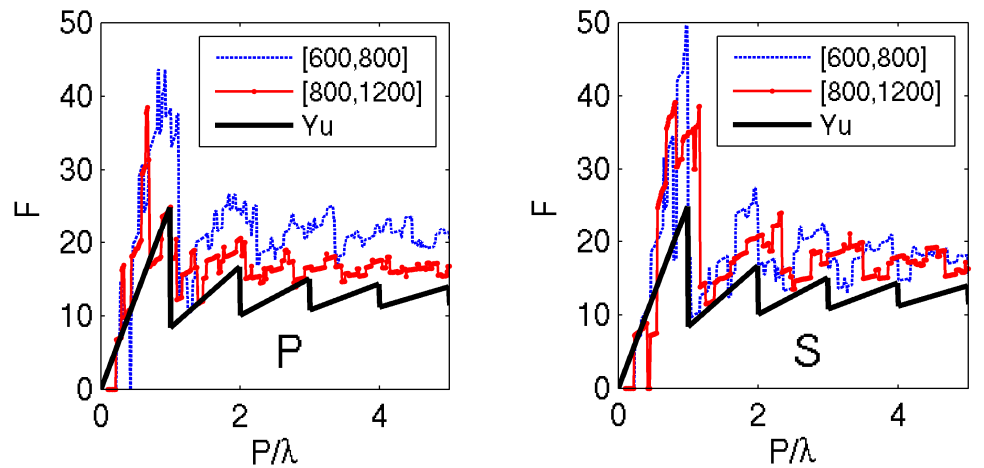

Figure 1- (Color online) Upper limit of absorption enhancement factor introduced by $1 \mathrm{D}$ gratings in $\mathrm{P}$ (a) and $\mathrm{S}$ polarization (b) for a slab with $\mathrm{d}=200 \mathrm{~nm}$ and $\mathrm{n}=4$. Blue dashed: averaged over [600 -800] nm. Solid: averaged over [800 -1200] nm. Bold solid: Yu's model.

The authors acknowledge funding by the Swiss National Science Foundation under project number 200020_137700/1.

\section{References}

[1] A. Shah, ed., Thin-film silicon solar cells, 1st ed. (EPFL Press, Lausanne, 2010).

[2] E. Yablonovitch and G. D. Cody, "Intensity enhancement in textured optical sheets for solar cells," IEEE Trans. Electron Devices 29, 300-305 (1982).

[3] H. R. Stuart and D. G. Hall, "Thermodynamic limit to light trapping in thin planar structures," JOSA A 14, 3001-3008 (1997).

[4] Z. Yu, A. Raman, and S. Fan, "Fundamental limit of nanophotonic light trapping in solar cells," PNAS 107, 17491-17496 (2010).

[5] Z. Yu, A. Raman, and S. Fan, "Fundamental limit of light trapping in grating structures," Opt. Express 18, A366-A380 (2010).

[6] F.-J. Haug, K. Söderström, A. Naqavi, and C. Ballif, "Resonances and absorption enhancement in thin film silicon solar cells with periodic interface texture," J. Appl. Phys. 109, 084516 (2011). 